\title{
Uma Comparação entre o Método dos Elementos de Contorno e o Método dos Elementos Finitos em Problemas de Campo Escalar Ortotrópicos
}

\author{
Laquini, R. ${ }^{1 *}$; Loeffler, C.F. ${ }^{1 *} ;$ Pinheiro, V. P. ${ }^{2 *}$ \\ 1 Programa de Pós-Graduação em Engenharia Mecânica, Universidade Federal do Espírito Santo, Vitória, ES, Brasil. \\ 2 Departamento de Engenharia Mecânica, MULTIVIX, Vitória, ES, Brasil. \\ * e-mail: raphaellaquini@hotmail.com \\ *e-mail: carlosloeffler@bol.com.br \\ * e-mail: vitor.pinheiro1987@gmail.com
}

\begin{abstract}
Resumo
Cada vez mais a simulação numérica tem sido utilizada como ferramenta de trabalho na engenharia e ciências afins. Curiosamente, embora o Método dos Elementos de Contorno (MEC) seja uma técnica discreta que se adapta facilmente a domínios não regulares e apresenta elevada precisão na simulação de problemas escalares em geral, não tem sido aplicado ostensivamente em problemas ortotrópicos, circunscrevendo-se a um limitado conjunto de aplicações em barragens. Usualmente, são adotadas técnicas que se baseiam na discretização do domínio, como o Método dos Elementos Finitos (MEF) e o Método dos Volumes Finitos. Almejando um maior alcance do MEC neste campo, este trabalho apresenta sua formulação e aplicação em problemas bidimensionais ortotrópicos, homogêneos e estacionários, que incluem comparações com o MEF. O objetivo é permitir uma melhor valoração do desempenho do MEC e ampliar suas perspectivas neste tipo de aplicação.
\end{abstract}

Palavras chave: Método dos Elementos de Contorno, Método dos Elementos Finitos, Problemas Ortotrópicos.

\begin{abstract}
Increasingly, the numerical simulation has been used as a tool in engineering and related sciences. Curiously, although the Boundary Element Method (BEM) is a discrete technique that adapts easily to nonregular domains and shows high accuracy in simulation of scalar problems in general, it has not been applied ostensibly in orthotropic problems, confining itself to a limited set of applications in dams. Domain discrete techniques such as the Finite Element Method (FEM) and the Finite Volumes are used preferentially. Craving to reach a greater wide spread to the BEM in this field, this paper presents its formulation and application in orthotropic two-dimensional, homogeneous and stationary problems, including comparisons with the FEM. The goal is to allow a better evaluation of the BEM performance and broaden their perspectives in this type of application.
\end{abstract}

Keywords: Boundary Elements Method, Finite Elements Method, Orthotropic Problems.

\section{Introdução}

Problemas de campo escalar ortotrópicos são bastante importantes na engenharia, pois muitas situações práticas requerem a distribuição de temperaturas e fluxos em materiais sintetizados, celulose, espumas e materiais porosos. Nesse último caso, uma vez que os modelos de difusividade hidráulica baseados na Equação de Darcy para simulação do fluxo têm sido amplamente usados na engenharia de petróleo, visando à extração de óleos em rochas, o modelo matemático resultante também se enquadra no contexto da Equação de Campo Escalar Generalizada [1], tal como ocorre nos problemas térmicos.

O Método dos Elementos de Contorno (MEC) tem facilidades de adaptação em domínios não regulares, contudo, seu modelo ortotrópico não tem recebido destaque. Portanto, este trabalho tem por objetivo mostrar o funcionamento do MEC em comparação com 
Método do Elementos Finitos (MEF) em problemas de solução analítica conhecida.

\section{Equação de Governo}

Considere domínios bidimensionais com propriedades homogêneas, na condição de estado estacionário e sem fontes ou sorvedouros. Sob essas hipóteses simplificadoras, a Equação de Campo Escalar Generalizada é dada por:

$$
\nabla \cdot[\mathrm{K} \nabla u(\mathrm{X})]=0
$$

Na Eq. (1), $\nabla$ é o operador Nabla, $u(X)$ é a variável básica ou potencial, $\mathrm{X}=\mathrm{X}\left(x_{j}\right)$ e $\mathrm{K}$ é um diádico, no qual figuram as propriedades constitutivas do meio.

Em duas dimensões, $\mathrm{K}$ apresenta-se com quatro coeficientes distintos, representando um material dito anisotrópico. Contudo, tomando-se o sistema coordenado em coincidência com as direções cristalográficas, pode-se simplificar o tratamento matemático de casos importantes, pois alguns materiais apresentam orientações cristalinas mais simples, como o sistema cristalino ortorrômbico, em que o tensor $\mathrm{K}$ é simétrico. Assim, a equação de governo pode ser escrita simplesmente como:

$$
k_{1} \frac{\partial^{2} u}{\partial x_{1}^{2}}+k_{2} \frac{\partial^{2} u}{\partial x_{2}^{2}}=0
$$

A figura 1 ilustra algumas características importantes do modelo físico, particularmente o domínio $\Omega$, delimitado pelo contorno $\Gamma . \Gamma_{u}$ e $\Gamma_{q}$ representam as fronteiras com condições de contorno essencial e natural, respectivamente. A condição de contorno essencial ou de Dirichlet é aquela em que o potencial $u(\mathrm{X})$ é prescrito, já a condição de contorno natural ou de Neumann ocorre quando a derivada do potencial com relação à direção normal externa unitária $n=n(X)$ é imposta. $k_{1}$ e $k_{2}$ são as constantes de difusividade nas direções de ortotropia $x_{1}$ e $x_{2}$, respectivamente.

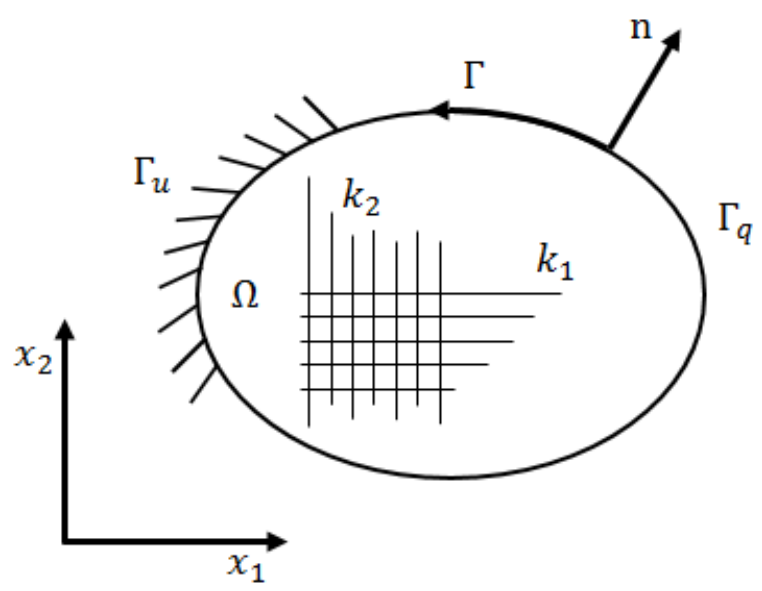

Figura 1: Modelo físico genérico de um problema ortotrópico.

\section{Método dos Elementos de Contorno}

Pode-se formular a equação integral do MEC escrevendo-se a forma integral forte associada à equação diferencial de governo [2], ou seja:

$$
\int_{\Omega}\left[k_{1} \frac{\partial^{2} u(\mathrm{X})}{\partial x_{1}^{2}} u^{*}+k_{2} \frac{\partial^{2} u(\mathrm{X})}{\partial x_{2}^{2}} u^{*}\right] d \Omega(\mathrm{X})=0
$$

No caso do MEC, a função auxiliar $u^{*}=u^{*}(\xi ; \mathrm{X})$ é a solução fundamental, que corresponde à solução de um problema correlato com domínio infinito, no qual uma fonte concentrada é aplicada num ponto $\xi$ particular.

Aplicando-se a regra do produto de funções duas vezes no núcleo da integral da Eq. (3) chega-se a:

$$
\begin{gathered}
\int_{\Omega}-u\left\{\left[\frac{\partial}{\partial x_{1}}\left(k_{1} \frac{\partial u^{*}}{\partial x_{1}}\right)\right]+\left[\frac{\partial}{\partial x_{2}}\left(k_{2} \frac{\partial u^{*}}{\partial x_{2}}\right)\right]\right\} d \Omega+ \\
\int_{\Omega} u^{*}\left\{\left[\frac{\partial}{\partial x_{1}}\left(k_{1} \frac{\partial u}{\partial x_{1}}\right)\right]+\left[\frac{\partial}{\partial x_{2}}\left(k_{2} \frac{\partial u}{\partial x_{2}}\right)\right]\right\} d \Omega+\int_{\Omega} u\left(k_{1} \frac{\partial^{2} u^{*}}{\partial x_{1}^{2}}+\right. \\
\left.k_{2} \frac{\partial^{2} u^{*}}{\partial x_{2}^{2}}\right) d \Omega=0
\end{gathered}
$$

Usando-se então o Teorema da Divergência, obtém-se a denominada forma integral inversa [3]:

$$
\begin{gathered}
\int_{\Gamma}\left[-u\left(k_{1} \frac{\partial u^{*}}{\partial x_{1}} \mathrm{n}_{x_{1}}+k_{2} \frac{\partial u^{*}}{\partial x_{2}} \mathrm{n}_{x_{2}}\right)+u^{*}\left(k_{1} \frac{\partial u}{\partial x_{1}} \mathrm{n}_{x_{1}}+\right.\right. \\
\left.\left.k_{2} \frac{\partial u}{\partial x_{2}} \mathrm{n}_{x_{2}}\right)\right] d \Gamma+\int_{\Omega} u\left(k_{1} \frac{\partial^{2} u^{*}}{\partial x_{1}^{2}}+k_{2} \frac{\partial^{2} u^{*}}{\partial x_{2}^{2}}\right) d \Omega=0
\end{gathered}
$$

Para se eliminar a integral de domínio restante na Eq. (5), é importante operar a seguinte alteração de coordenadas [4]:

$$
z_{i}=\frac{x_{i}}{\sqrt{k_{i}}}
$$

Então, substituindo-se a Eq. (6) no problema fundamental e expressando a fonte concentrada em termos de duas variáveis [5], obtém-se:

$$
\frac{\partial^{2} u^{*}}{\partial z_{1}^{2}}+\frac{\partial^{2} u^{*}}{\partial z_{2}^{2}}=-\Delta\left(\xi ; z_{1} \sqrt{k_{1}}\right) \Delta\left(\xi ; z_{2} \sqrt{k_{2}}\right)
$$

A Eq. (7) é semelhante ao problema fundamental de Laplace, mas representa um problema correlato ortotrópico. Resolvendo-a tem-se:

$$
\begin{aligned}
& u^{*}=\frac{1}{2 \pi \sqrt{k_{1} k_{2}}} \ln \frac{1}{\left(\frac{x_{1}^{2}}{k_{1}}+\frac{x_{2}^{2}}{k_{2}}\right)^{1 / 2}} \\
& q^{*}=\frac{-1}{2 \pi \sqrt{k_{1} k_{2}}} \frac{x_{1} \mathrm{n}_{x 1}+x_{2} \mathrm{n}_{x 2}}{\left(\frac{x_{1}^{2}}{k_{1}}+\frac{x_{2}^{2}}{k_{2}}\right)}
\end{aligned}
$$

Substituindo a Eq. (7) na integral de domínio existente na Eq. (5) e aplicando-se as propriedades do Delta de Dirac, obtém-se a seguinte equação integral [6]: 
$c(\xi) u(\xi)+\int_{\Gamma}\left[u(\mathrm{X}) q^{*}(\xi ; \mathrm{X})-q(\mathrm{X}) u^{*}(\xi ; \mathrm{X})\right] d \Gamma=0$

Por simplicidade, fez-se:

$$
\begin{aligned}
& q=k_{1} \frac{\partial u}{\partial x_{1}} \mathrm{n}_{x_{1}}+k_{2} \frac{\partial u}{\partial x_{2}} \mathrm{n}_{x_{2}}=0 \\
& q^{*}=k_{1} \frac{\partial u^{*}}{\partial x_{1}} \mathrm{n}_{x_{1}}+k_{2} \frac{\partial u^{*}}{\partial x_{2}} \mathrm{n}_{x_{2}}=0
\end{aligned}
$$

O coeficiente $c(\xi)$ depende da posição do ponto fonte em relação ao domínio físico $\Omega(\mathrm{X})$ e se está localizado no interior, externamente ou sobre o contorno $\Gamma(\mathrm{X})$. Neste último caso, também depende da suavidade deste [6].

O próximo passo é a discretização, em que o contorno é dividido numa série de elementos, ao longo dos quais se define a variação do campo e da geometria. Neste trabalho aproximam-se linearmente o potencial e sua derivada normal por elementos de contorno retilíneos. Os pontos campo geram os pontos nodais, nos quais $u(\mathrm{X})$ e $q(\mathrm{X})$ são prescritos ou calculados. Para cada ponto fonte $\xi$, é feita uma integração ao longo dos elementos de contorno, gerando um sistema matricial na forma:

$$
\mathrm{Hu}=\mathrm{Gq}
$$

\section{Método dos Elementos Finitos}

O ponto de partida do MEF é similar ao do MEC. Escreve-se a forma integral forte associada à equação diferencial de governo, dada por:

$$
\int_{\Omega}\left[k_{1} \frac{\partial^{2} u(\mathrm{X})}{\partial x_{1}^{2}} w(\mathrm{X})+k_{2} \frac{\partial^{2} u(\mathrm{X})}{\partial x_{2}^{2}} w(\mathrm{X})\right] d \Omega(\mathrm{X})=0
$$

A principal diferença neste momento é que a função auxiliar não mais é a solução fundamental. No MEF a função $w(\mathrm{X})$ usualmente é um polinômio, que expressa uma variação admissível do potencial [7].

Assim, a partir de um desenvolvimento matemático simples, que envolve a aplicação da regra da derivada do produto de duas funções uma vez apenas, obtémse:

$$
\begin{aligned}
& \int_{\Omega}\left\{k_{1}\left[\frac{\partial}{\partial x_{1}}\left(\frac{\partial u}{\partial x_{1}} w\right)-\frac{\partial u}{\partial x_{1}} \frac{\partial w}{\partial x_{1}}\right]+k_{2}\left[\frac{\partial}{\partial x_{2}}\left(\frac{\partial u}{\partial x_{2}} w\right)-\right.\right. \\
& \left.\left.\frac{\partial u}{\partial x_{2}} \frac{\partial w}{\partial x_{2}}\right]\right\} d \Omega=0
\end{aligned}
$$

Em seguida, aplica-se o Teorema da Divergência para chegar à forma integral fraca, ponto de partida para o desenvolvimento do MEF clássico:

$$
\begin{aligned}
& \int_{\Gamma}\left(k_{1} \frac{\partial u}{\partial x_{1}} \mathrm{n}_{x_{1}}+k_{2} \frac{\partial u}{\partial x_{2}} \mathrm{n}_{x_{2}}\right) w d \Gamma-\int_{\Omega}\left(k_{1} \frac{\partial u}{\partial x_{1}} \frac{\partial w}{\partial x_{1}}+\right. \\
& \left.k_{2} \frac{\partial u}{\partial x_{2}} \frac{\partial w}{\partial x_{2}}\right) d \Omega=0
\end{aligned}
$$

Esta sentença é aplicada em cada partição do domínio, os elementos finitos. A última integral gera matrizes de rigidez locais que, para o caso ortotrópico, são dadas por:

$$
\mathrm{H}_{i j}^{e}=\int_{\Omega^{\mathrm{e}}}\left(k_{1} \frac{\partial N_{i}}{\partial x_{1}} \frac{\partial N_{j}}{\partial x_{1}}+k_{2} \frac{\partial N_{i}}{\partial x_{2}} \frac{\partial N_{j}}{\partial x_{2}}\right) d \Omega^{\mathrm{e}}(\mathrm{X})
$$

Os índices $i$ e $j$ se referem à possibilidade de interação entre os diferentes nós de um mesmo elemento, guardando a mesma ideia dos coeficientes de influência da mecânica estrutural e da relação entre pontos fonte $\xi$ e pontos campos X no caso do MEC. Contudo, ao contrário deste, a parte operacional do MEF é bem mais elaborada, porque não se trata de resolver integrais de linha, mas sim de realizar integrações sobre áreas elementares. No caso dos elementos triangulares aqui usados, é preciso fazer uma transformação de coordenadas para integração dessas áreas dos domínios elementares $\Omega^{\mathrm{e}}$ de modo que a descrição do potencial no interior dos elementos é dada por uma aproximação linear na forma:

$$
N_{i}=\frac{a_{i}+b_{i} x_{1}+c_{i} x_{2}}{2 \mathrm{~A}^{\mathrm{e}}}
$$

Nesta última equação, $a_{i}, b_{i}$ e $c_{i}$ são coeficientes da interpolação linear e $\mathrm{A}^{\mathrm{e}}$ é a área do elemento. É possível estabelecer uma aproximação para o potencial $u(\mathrm{X})$ em cada elemento como função dos pontos nodais do elemento [8]. Após laboriosos algebrismos, as matrizes de difusividade elementar no caso ortotrópico ficam compostas por duas partes:

$$
\begin{gathered}
H^{e}=\frac{k_{1}}{4 \mathrm{~A}^{\mathrm{e}}}\left[\begin{array}{lll}
b_{1} b_{1} & b_{1} b_{2} & b_{1} b_{3} \\
b_{2} b_{1} & b_{2} b_{2} & b_{2} b_{3} \\
b_{3} b_{1} & b_{3} b_{2} & b_{3} b_{3}
\end{array}\right]+ \\
\frac{k_{2}}{4 \mathrm{~A}^{\mathrm{e}}}\left[\begin{array}{lll}
c_{1} c_{1} & c_{1} c_{2} & c_{1} c_{3} \\
c_{2} c_{1} & c_{2} c_{2} & c_{2} c_{3} \\
c_{3} c_{1} & c_{3} c_{2} & c_{3} c_{3}
\end{array}\right]
\end{gathered}
$$

A integral de contorno mostrada na Eq. (16) também é tratada em nível local, gerando vetores elementares de fluxo [9]. Ao final, um sistema linear de equações é gerado pela montagem das matrizes elementares, que são conectadas através da condição de admissibilidade, garantindo a continuidade do potencial $u(\mathrm{X})$ através de valores nodais comuns entre os elementos:

$$
\mathrm{Hu}=\mathrm{f}
$$

\section{Simulações Numéricas}

\subsection{Primeiro Teste}

Um domínio quadrado, de lados unitários, é submetido a condições de contorno de Dirichlet e Neumann, conforme mostrado na Figura 2. Este problema pode ser interpretado de vários modos, segundo a 
conveniência. Pode ser encarado como uma chapa na qual se impõe um gradiente de temperaturas e fluxos de calor, mas também poderia ser fisicamente interpretado de como um domínio ou região porosa, submetida a um perfil de potenciais ou alturas piezométricas distintas.

Os resultados numéricos do MEC e do MEF foram comparados com o valor da solução analítica, obtida pelo Método da Separação de Variáveis. Os valores das difusividades escolhidos foram $k_{x}=1, k_{y}=0,25$; estes foram tomados próximos da unidade porque em problemas governados pela Equação de Laplace, apenas a relação entre as difusividades é importante.

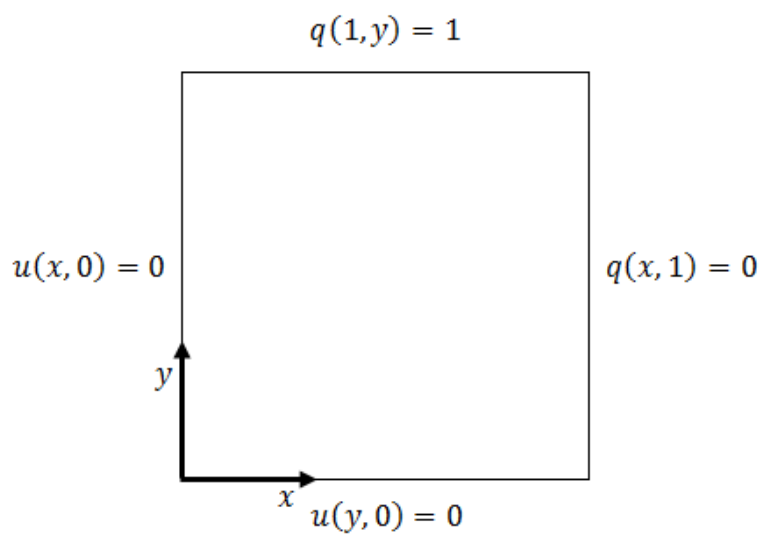

Figura 2: Chapa quadrada submetida a condições de temperaturas e fluxos prescritos.

No cálculo do erro relativo médio percentual, considerou-se o maior valor analítico do caso como denominador na medida de erro relativo em cada nó. $A$ figura 3 mostra os resultados do MEC e a figura 4 os resultados correspondentes para o MEF. Foram calculados os valores do potencial em diversos pontos internos. Foram escolhidos pontos com as mesmas coordenadas para comparação entre os métodos.

Neste caso, observa-se uma nítida vantagem do MEC, alcançando patamares de erro muito menores do que 0 MEF, para malhas com muito menos quantidade de pontos nodais. Destaca-se, particularmente, o reduzido valor do erro já alcançado com malhas pouco refinadas.

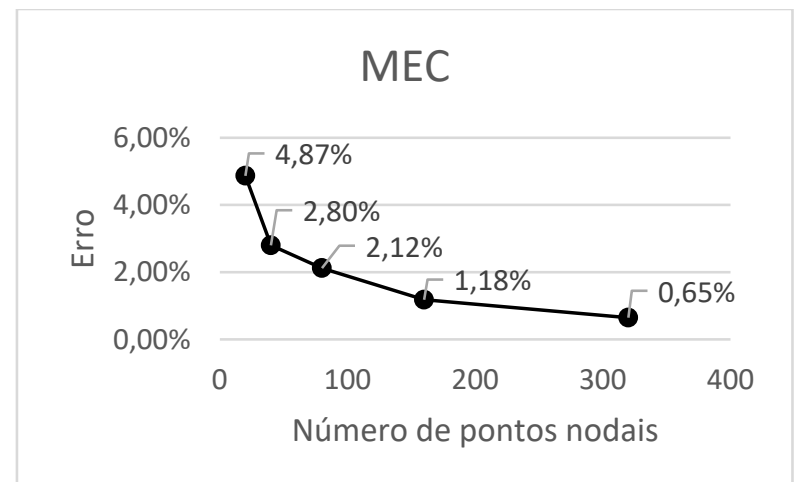

Figura 3: Curva de erro médio percentual para potencial com o MEC.

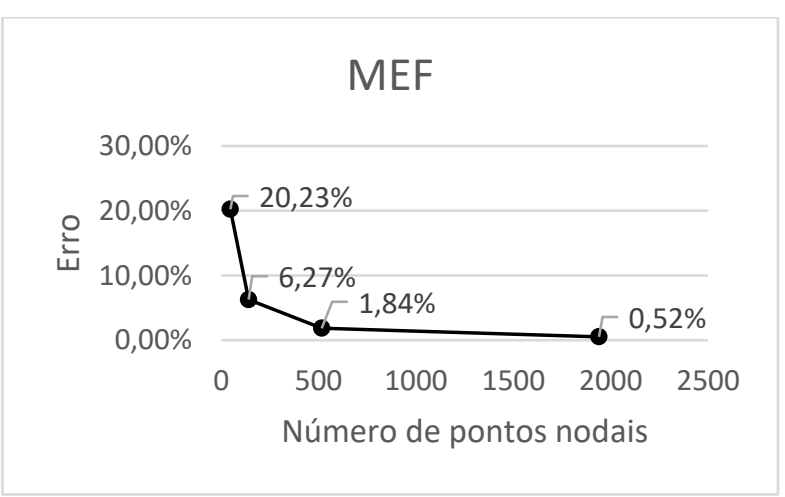

Figura 4: Curva de erro médio percentual para potencial com o MEF.

\subsection{Segundo Teste}

No segundo teste, a mesma chapa é submetida exclusivamente a condições de Dirichlet, conforme apresentado na figura 5 .

Há descontinuidades entre os potenciais nos vértices superior esquerdo e inferior direito, induzindo a fluxos elevadíssimos nas regiões próximas à singularidade. Tal situação é irreal na prática, mas serve para testar a robustez do método, particularmente o MEC, que lida com os valores descontínuos em seu sistema de equações. Já o MEF, pela sua formulação matemática, elimina as linhas e colunas do sistema matricial que são referentes às condições de potencial, amenizando $o$ efeito das descontinuidades.

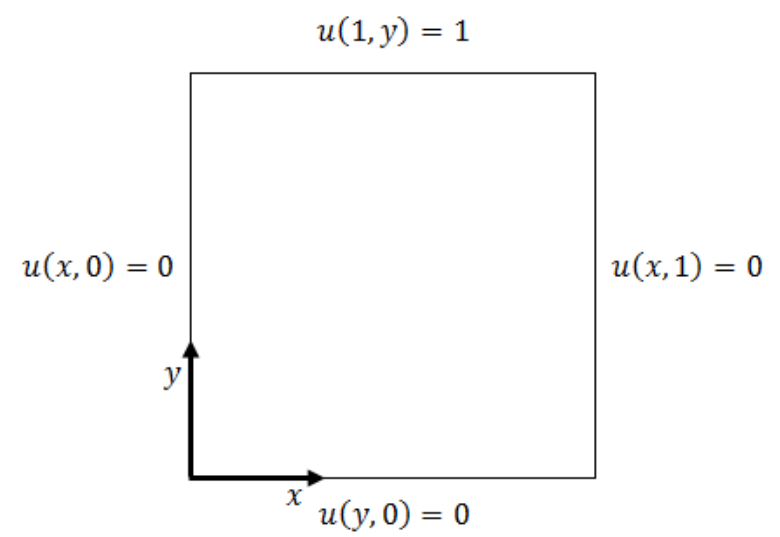

Figura 5: Chapa quadrada submetida a condições de temperaturas prescritos

Além disso, ressalta-se que sendo $k_{y}$ quatro vezes menor do que $k_{x}$ e estando os fluxos de calor direcionados na direção vertical, o problema torna-se numericamente bem mais difícil.Os resultados são mostrados nas figuras 6 para o MEC e 7 para o MEF.

De fato, os valores do erro cresceram bastante no MEC, mas sua curva de erro apresentou a monotonicidade devida e a faixa de erro percentual para a sua malha mais refinada foi similar a alcançada pelo MEF para a mesma quantidade de pontos nodais. 


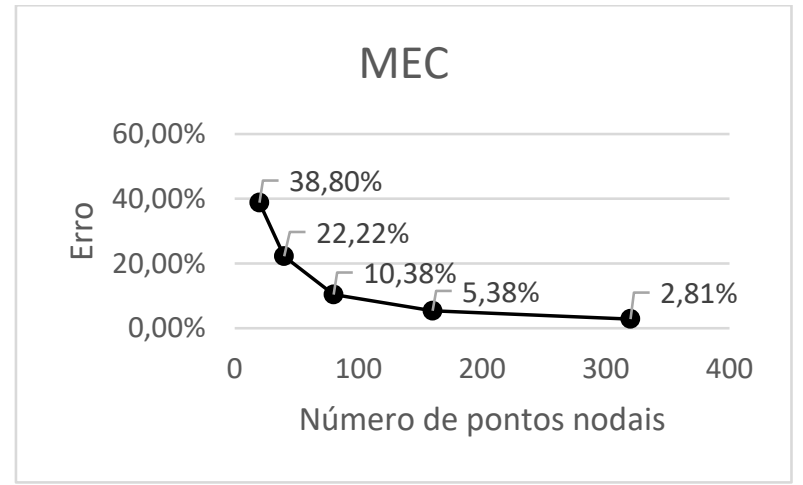

Figura 6: Curva de erro médio percentual para potencial com o MEC.

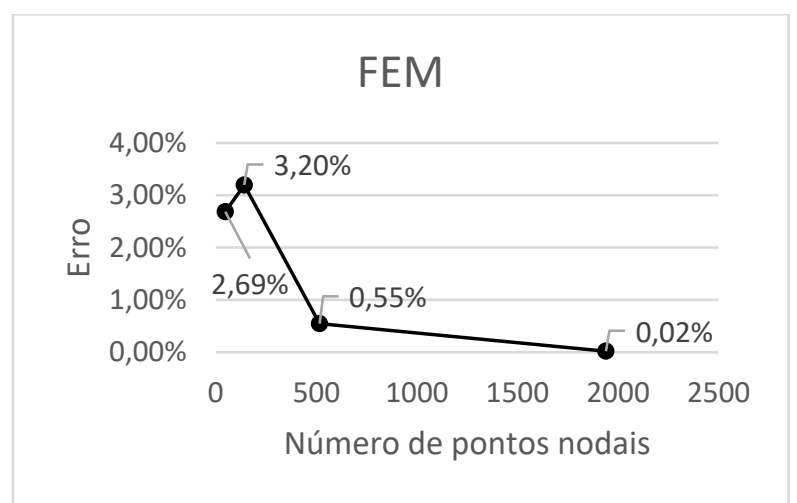

Figura 7: Curva de erro médio percentual para potencial com o MEF.

Vale ressaltar que em MEC os potenciais no interior do domínio são calculados em função dos potenciais e fluxos do contorno e, como dito anteriormente, as descontinuidades geram fluxos elevadíssimos o que contribui para a distorção dos resultados. No caso do MEF, apesar de pequena oscilação na curva de erro para as malhas menos refinadas, o desempenho geral foi superior ao apresentado no primeiro caso.

\section{Conclusões}

O desempenho do MEC foi bem superior ao apresentado pelo MEF no caso em que figuravam condições de Dirichlet e Neumann em conjunto, que são as condições usuais nos problema práticos de engenharia. Já no caso em que houve descontinuidades resultantes da aplicação exclusiva de condições de Dirichlet, apresentou um desempenho discretamente inferior ao MEF, pelo fato dos valores internos serem calculados a partir de potenciais e fluxos calculados no contorno. Contudo, mesmo neste teste, propositadamente abordado para avaliar sua robustez numérica, o MEC mostrou redução contínua de erros e resultados bastante aceitáveis. Também é oportuno ressaltar que o modelo numérico referente ao MEC não empregou qualquer técnica de solução de integrais quase-singulares, que certamente melhoraria o desempenho do método, uma vez que o argumento da solução fundamental é afetado pelo valor das propriedades de ortotropia material.

\section{Agradecimentos}

Os autores agradecem a FAPES pelo apoio financeiro.

\section{Referências}

[1] LOEFFLER, C. F. Modelos Mecânicos Derivados da Equação de Campo Escalar Generalizada, Revista Militar de Ciência e Tecnologia, Biblioteca do Exército. vol. 4, n. 1, pp. 24-38, 1992.b.

[2] BREBBIA, C. A., WALKER, S., Boundary Element Techniques in Engineering, Newnes-Butterworths: London, 1980.

[3] BREBBiA, C. A., TElles, J. C., WROBEL, L. C. Boundary Element Techniques. Springer Verlag, 1984.

[4] PEREZ, M. M., WROBEL, L. C. A General Integral Equation Formulation for Homogeneous Ortotropic Potential Problems, Eng. An. Bound. Elem, 10, 323332, 1992.

[5] RAISINGHANIA, M. D. Integral Equations and Boundary Value Problems, S. Chand Ed., New Dehli, 2011.

[6] BREBBIA, C. A., DOMINGUEZ, J. Boundary Elements - An Introductory Course. WIT Press, 1992.

[7] ZIENKIEWCZ, O., TAYLOR, R. The Finite Element Method, McGraw Hill, 5th Edition, 1989.

[8] REDDY, J. An Introduction to the Finite Element Method, McGraw-Hill, 3rd edition, 2005.

[9] BATHE, K. J., Finite Element Procedures, PrenticeHall: New Jersey, 1995. 